\title{
Surface plasmon resonance biosensor for exosome detection based on reformative tyramine signal amplification activated by molecular aptamer beacon
}

Wenqin Chen ${ }^{1,2 \dagger}$, Zhiyang $\mathrm{Li}^{2 \dagger}{ }^{\text {, Wenqian Cheng }}{ }^{1}$, Tao $\mathrm{Wu}^{3}$, Jia Li ${ }^{1}$, Xinyu Li ${ }^{1}, \mathrm{Lin} \mathrm{Liu}^{3}$, Huijie Bai ${ }^{1}$, Shijia Ding ${ }^{1}$, Xinmin $\mathrm{Li}^{1 *}$ and Xiaolin $\mathrm{Yu}^{3^{*}}$

\begin{abstract}
Human epidermal growth factor receptor 2 (HER2)-positive exosomes play an extremely important role in the diagnosis and treatment options of breast cancers. Herein, based on the reformative tyramine signal amplification (TSA) enabled by molecular aptamer beacon (MAB) conversion, a label-free surface plasmon resonance (SPR) biosensor was proposed for highly sensitive and specific detection of HER2-positive exosomes. The exosomes were captured by the HER2 aptamer region of MAB immobilized on the chip surface, which enabled the exposure of the G-quadruplex DNA (G4 DNA) that could form peroxidase-like G4-hemin. In turn, the formed G4-hemin catalyzed the deposition of plentiful tyramine-coated gold nanoparticles (AuNPs-Ty) on the exosome membrane with the help of $\mathrm{H}_{2} \mathrm{O}_{2}$, generating a significantly enhanced SPR signal. In the reformative TSA system, the horseradish peroxidase (HRP) as a major component was replaced with nonenzymic G4-hemin, bypassing the defects of natural enzymes. Moreover, the dual-recognition of the surface proteins and lipid membrane of the desired exosomes endowed the sensing strategy with high specificity without the interruption of free proteins. As a result, this developed SPR biosensor exhibited a wide linear range from $1.0 \times 10^{4}$ to $1.0 \times 10^{7}$ particles $/ \mathrm{mL}$. Importantly, this strategy was able to accurately distinguish HER2-positive breast cancer patients from healthy individuals, exhibiting great potential clinical application.
\end{abstract}

Keywords: HER2-positive exosomes, Surface plasmon resonance, Tyramine signal amplification, G4-hemin, Breast cancer diagnosis

\footnotetext{
*Correspondence: xinmnli@163.com; yuxiaolincq@hotmail.com

tWenqin Chen and Zhiyang Li contributed equally to this work

${ }^{1}$ Key Laboratory of Clinical Laboratory Diagnostics (Ministry

of Education), College of Laboratory Medicine, Chongqing Medical

University, Chongqing 400016, China

${ }^{3}$ Department of Laboratory Medicine, Zigong Fourth People's Hospital,

Sichuan 643000, China

Full list of author information is available at the end of the article
}

(c) The Author(s) 2021. Open Access This article is licensed under a Creative Commons Attribution 4.0 International License, which permits use, sharing, adaptation, distribution and reproduction in any medium or format, as long as you give appropriate credit to the original author(s) and the source, provide a link to the Creative Commons licence, and indicate if changes were made. The images or other third party material in this article are included in the article's Creative Commons licence, unless indicated otherwise in a credit line to the material. If material is not included in the article's Creative Commons licence and your intended use is not permitted by statutory regulation or exceeds the permitted use, you will need to obtain permission directly from the copyright holder. To view a copy of this licence, visit http://creativecommons.org/licenses/by/4.0/. The Creative Commons Public Domain Dedication waiver (http://creativecommons.org/publicdomain/zero/1.0/) applies to the data made available in this article, unless otherwise stated in a credit line to the data. 


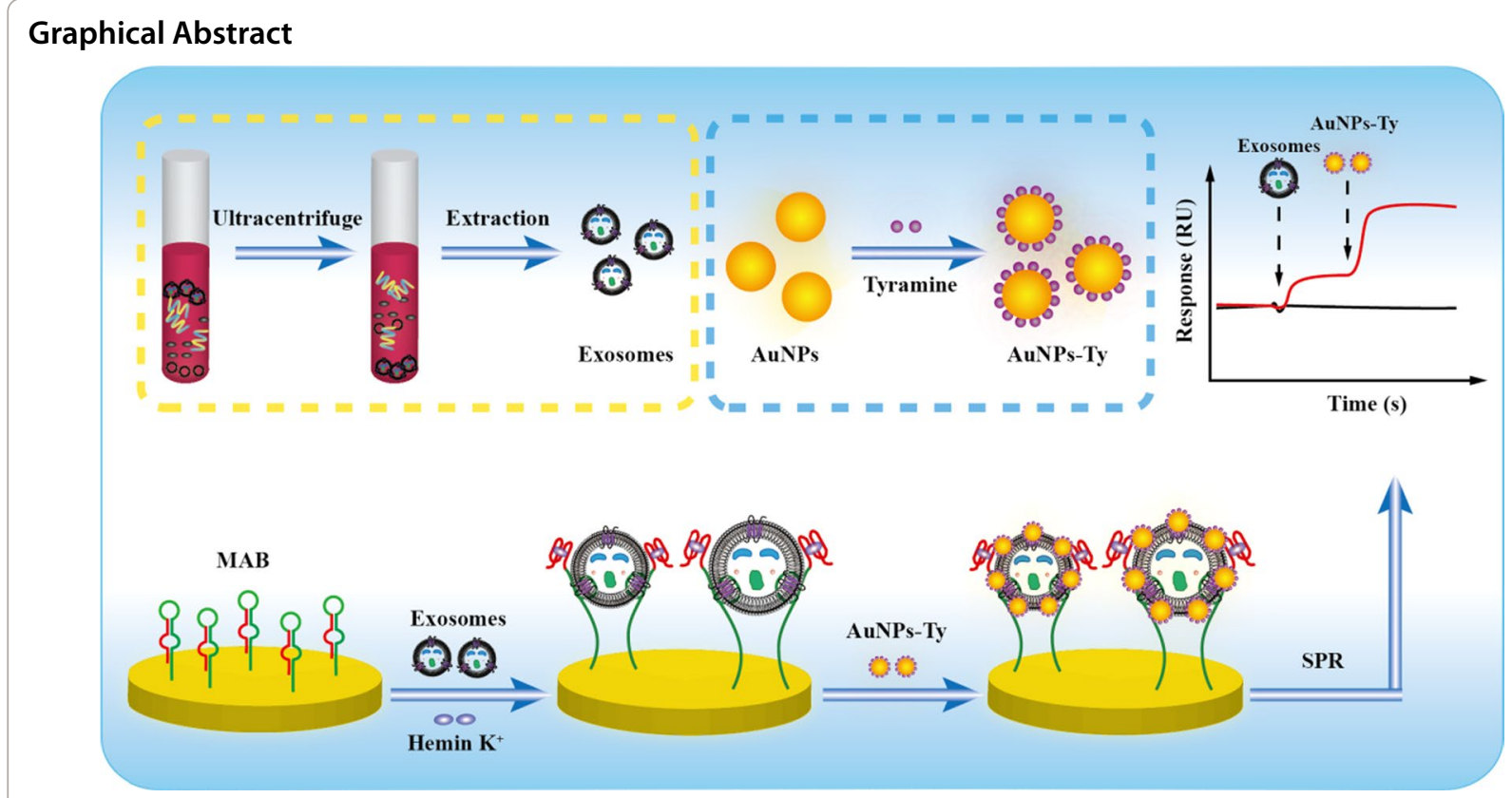

\section{Introduction}

Breast cancer is the most common type of cancer that causes female deaths worldwide [1, 2]. In the diagnosis and treatment process of breast cancer, human epidermal growth factor receptor 2 (HER2) is an important prognostic indicator and predictor of HER2-targeted drugs $[3,4]$. Currently, the routine methods for HER2 analysis mainly include immunohistochemistry and fluorescence in situ hybridization, but these methods have several shortcomings, such as the uncertainty of the results, the high false-positive rates, and the excessively harsh requirements for specimens, etc. [5]. Therefore, developing a noninvasive and reliable strategy to accurately monitor HER2 status is challenging but urgently needed.

Exosomes are $30-150 \mathrm{~nm}$ sized extracellular vesicles with a typical lipid bilayer membrane structure, which are secreted into the surrounding biofluids by different types of cells $[6,7]$. In particular, tumor-derived exosomes not only carry cancer-specific proteins but have high abundance and stability in body fluids, thus acting as promising biomarkers for liquid biopsy [8]. Recent reports have proved that the HER2-positive exosomes are greatly increased in patients with breast cancer, and there is a good consistency of the HER2 expression level between exosomes and tumor tissues $[9,10]$. Therefore, quantitating the amounts of HER2-positive exosomes has the potential to improve the diagnosis and customize treatment of breast cancer.

Apart from traditional methods including nanoparticle tracking analysis (NTA), western blot, flow cytometry, and enzyme-linked immunosorbent assay (ELISA) [11], various biosensing platforms have been developed for exosomes analysis by targeting their surface proteins using the corresponding antibodies or aptamers [12-17]. Among them, surface plasma resonance (SPR) biosensor is receiving extensive attention because it is a rapid, real-time, and label-free diagnostic device $[18,19]$. However, SPR biosensor for exosomes detection faces adverse conditions: (i) the small size and low mass of exosomes cannot trigger obvious signal discrepancy, leading to the inevitable requirement of signal amplifier, and (ii) the collected exosomes are always mingled with free target proteins from serum, resulting in the generation of a false-positive signal that reduces the accuracy of results. Considering these facts, we are spurred to develop a novel SPR biosensing strategy for sensitive and specific detection of HER2-positive exosomes.

Tyramine signal amplification (TSA) is a typical enzyme-assisted amplification strategy in which tyramine can be transformed by horseradish peroxidase (HRP) into a reactive oxidized intermediate with the help of $\mathrm{H}_{2} \mathrm{O}_{2}$ [20]. The intermediate then covalently conjugates and rapidly deposits on near protein residues to trigger signal enhancement $[21,22]$. Recently, our group proposed a cytosensor that used HRP to catalyze the deposition of tyramine-modified electroactive reporters on tumor cells, exhibiting significantly enhanced sensitivity [23]. Nevertheless, HRP has suffered from complicated purification processes and poor chemical stability due to the intrinsic characters of nature enzymes [24], which 
compromise the clinical applicability of TSA-based strategies. G-quadruplex-hemin (G4-hemin), an HRP-mimicking DNAzyme with excellent catalytic performance, has been widely applied in biosensing owing to its easy synthesis, high stability, and low requirement for reaction conditions $[25,26]$. Therefore, G4-hemin could be a credible and promising alternative to HRP.

Herein, a label-free SPR biosensor was proposed for highly sensitive and specific detection of HER2-positive exosomes based on reformative TSA activated by targetinduced molecular aptamer beacon (MAB) conversion. By combining the functions of G4 and HER2 aptamer into one molecular beacon, the engineered MABs immobilized on the sensing chip bound to HER2-positive exosomes, resulting in the structural change that accompanied the exposure of G4. Then, the formed G4-hemin catalyzed the deposition of numerous tyramine-coated gold nanoparticles (AuNPs-Ty) on the lipid membrane of the exosomes in the presence of $\mathrm{H}_{2} \mathrm{O}_{2}$, achieving a geometrically enhanced SPR signal. Profiting from the superior catalytic ability of G4-hemin, the improved TSA had no need for HRP. What's more, the dual-identification of surface proteins and the membrane structure of exosomes was able to avoid the interference of the free proteins. Taken together, the developed SPR biosensing strategy enabled accurate detection of HER2-positive exosomes with high sensitivity and specificity.

\section{Experimental}

\section{Reagents and materials}

Sodium citrate powder, $\mathrm{HAuCl}_{4} \cdot 4 \mathrm{H}_{2} \mathrm{O}$ and 11-mercaptoundecanoic acid (MUA) were purchased from Sinopharm Chem Co., Ltd (Shanghai, China). Hemin, tyramine, and 6-Mercapto-1-hexanol $(\mathrm{MCH})$ were purchased from Sigma-Aldrich (St. Louis, USA). Hemin was dissolved in dimethyl sulfoxide as the stock solution and then diluted to the required concentration with $25 \mathrm{mM}$ HEPES buffer ( $\mathrm{pH}$ 7.4, $25 \mathrm{mM}$ HEPES, $200 \mathrm{mM} \mathrm{NaCl}$, $100 \mathrm{mM} \mathrm{KCl}, 1 \%$ DMSO, 0.05\% Triton). Fetal Bovine Serum (FBS) and Dulbecco's Modified Eagle Medium (DMEM) were offered by Gibco (Gaithersburg, USA). $\mathrm{N}$-hydroxysuccinimide (NHS) and $\mathrm{N}$-(3-(dimethylamino) propyl)-N'-ethylcarbodiimide hydrochloride (EDC) were purchased from Alfa Aesar (Massachusetts, USA). Phosphate buffer (PBS) was supplied by Thermo Fisher Scientific (Wilmington, USA). All the chemicals used were of analytical reagent grade, and all the HPLC-purified oligonucleotides listed in Additional file 1: Table S1 were prepared by Sangon Biotech. Co., Ltd (Shanghai, China). Tris-EDTA (TE) buffer (10 mM Tris-HCl, 1 mM EDTA, $\mathrm{pH}$ 8.0) was utilized for the dissolution of oligonucleotides. Aqueous solutions prepared by Millipore Milli-Q gradient ultrapure water system were used (Millipore Co.,
MA, USA). Clinical samples were collected from the First Affiliated Hospital of Chongqing Medical University.

\section{Instruments}

All measurements were carried out on the SPR biochemical analyzer independently developed and built by our group. The sensing principle of this technique is to measure the change in refractive index around the surface of the metallic sensor chip due to alterations in mass caused by analyte-receptor noncovalent interactions $[27,28]$. The SPR analyzer with a signal acquisition frequency of $5 \mathrm{~s}$ is mainly composed of a light source, a sensing chip, a flow cell, and a CCD detector. The sensorgrams are analyzed by the lab-developed program written in LabVIEW, exhibiting a time course of resonance units (RU). Transmission electron microscopic (TEM) image was performed with an H-7500 transmission electron microscope (Hitachi High-Technologies Co., Japan). Nanoparticles tracking analysis (NTA) was supported by ZetaView (Particle Metrix, Germany). UV-Visible absorption spectra were carried out at a UV-2550 spectrophotometer (Shimadzu, Japan).

\section{Extraction of exosomes}

All cell lines, including HER2-positive SK-BR3 and HER2-negative HeLa, LNCaP, HepG2, and MCF-7 [2932], were provided by the American Type Culture Collection (ATCC) (Rockville, USA). DMEM (added with $10 \%$ FBS, $1 \%$ streptomycin and penicillin) was used for all kinds of cell culture in a sterile incubator containing 5\% $\mathrm{CO}_{2}$, at $37^{\circ} \mathrm{C}$. Cell passaging was processed when growth density reached $80 \%$. Ultimately, the cells were starved with serum-free medium for $48 \mathrm{~h}$ before the exosomes were extracted.

The exosomes were extracted from the cell culture medium according to the published works [33, 34]. First of all, the cell culture supernatant was collected and centrifuged $(10,000 \times g, 30 \mathrm{~min})$. This pretreatment process could separate the exosomes from larger vesicles and cell debris for preliminary purification. After centrifugation, the supernatant was collected and centrifuged again $(100,000 \times g, 70 \mathrm{~min})$ to extract the exosomes secreted by tumor cells. After that, the obtained exosomes were washed with $1 \times$ PBS to remove protein impurities, and centrifuged again for further purification $(100,000 \times g$, $70 \mathrm{~min}$ ). After that, the obtained exosomes were resuspended in $100 \mu \mathrm{L}$ of PBS and stored at $-80^{\circ} \mathrm{C}$ for later use. The extraction procedures of exosomes derived from serum are as follows. To begin with, $1 \mathrm{~mL}$ of PBS was added to $1 \mathrm{~mL}$ of serum and then centrifuged $(12,000 \times g$, $45 \mathrm{~min})$. Afterward, the obtained supernatant was centrifuged again $(110,000 \times g, 2 \mathrm{~h})$. After that, the supernatant was discarded, and the exosomes precipitated at 
the bottom were suspended in $8 \mathrm{~mL}$ of PBS solution and passed through a $0.22 \mu \mathrm{m}$ filter membrane to remove impurities with smaller particle diameters. Thereafter, the filtrate was centrifuged again $(110,000 \times g, 70 \mathrm{~min})$ for further purification. Finally, the pure exosomes obtained after centrifugation were resuspended in $100 \mu \mathrm{L}$ of PBS and stored at $-80{ }^{\circ} \mathrm{C}$ for later use. TEM and NTA were performed to characterize the obtained cancer cellderived exosomes.

\section{Preparation of AuNPs-Ty complex}

Firstly, AuNPs with a particle size of about $14 \mathrm{~nm}$ were synthesized according to the published works with slight modification [35-39]. Briefly, $88.2 \mathrm{~mL}$ of $\mathrm{HAuCl}_{4}(1 \mathrm{mM})$ was boiled and then added rapidly to $10.1 \mathrm{~mL}$ of sodium citrate $(34 \mathrm{mM})$. After the color changed to burgundy, the heating was stopped, and the mixture was cooled to room temperature with continued stirring. Then the prepared AuNPs and tyramine were combined by MUA that has sulfhydryl and carboxyl groups at each end. In detail, the sulfhydryl groups at one end of MUA were connected to AuNPs through $\mathrm{Au}-\mathrm{S}$ bonds, and the other end was connected to tyramine through carboxyl groups, which indirectly connected AuNPs and tyramine into AuNPsTy complex. To begin with, MUA $(25 \mu \mathrm{L}, 100 \mu \mathrm{M})$ was added to the AuNPs $(5 \mathrm{~mL})$ solution, gently shaking for $12 \mathrm{~h}$, and then the solution was centrifuged twice $(2000 \times g, 10 \mathrm{~min})$ to get AuNPs-MUA solution. Thereafter, NHS $(10 \mu \mathrm{L}, 1 \mathrm{mg} / \mathrm{mL})$, EDC $(10 \mu \mathrm{L}, 2 \mathrm{mg} / \mathrm{mL})$ and tyramine $(10 \mu \mathrm{L}, 100 \mu \mathrm{M})$ were added to the mixed solution, gently shaking for $12 \mathrm{~h}$ to get AuNPs-Ty complex, which was stored at $4{ }^{\circ} \mathrm{C}$ for later use.

\section{Exosomes analysis by the fabricated SPR biosensor}

Firstly, the gold chip and the flow cell were immersed in $75 \%$ ethanol, followed by sonication for $20 \mathrm{~min}$ at room temperature. Then the chip was rinsed with deionized water and blew dry with nitrogen. Next, the piranha solution $1 \mathrm{~mL}\left(\mathrm{H}_{2} \mathrm{SO}_{4}: 700 \mu \mathrm{L}, \mathrm{H}_{2} \mathrm{O}_{2}: 300 \mu \mathrm{L}\right)$ was freshly prepared, after cooling to room temperature, we covered it on the chip surface for $10 \mathrm{~min}$ to remove the remaining impurities. Again, the chip was rinsed thoroughly with deionized water and blew dry with nitrogen. Next, the capture probes of MAB were diluted to a concentration of $1.5 \mu \mathrm{M}$ by the fixative solution $\left(\mathrm{KH}_{2} \mathrm{PO}_{4}, 1 \mathrm{M}\right)$, and then modified on the surface of the chip overnight at $4{ }^{\circ} \mathrm{C}$. After the incubation, $1 \mathrm{mM} \mathrm{MCH}$ was used to block the excess binding sites for $30 \mathrm{~min}$. After washing and drying, the treated chip, the prism cleaned with $75 \%$ ethanol, and the flow cell were installed on the SPR instrument for subsequent testing. Thereafter, the flow pipeline was rinsed with cell grade $1 \times \mathrm{PBS}$ for $15 \mathrm{~min}$ to output the baseline signal (flow rate: $50 \mu \mathrm{L} / \mathrm{min}$ ). Then, different concentrations of exosomes derived from tumor cells or clinical samples were loaded (loading volume: $150 \mu \mathrm{L}$, flow rate: $7 \mu \mathrm{L} / \mathrm{min}$, time: $30 \mathrm{~min}$ ). Subsequently, after the signal was stabilized, the AuNPs-Ty composite was loaded (loading volume: $150 \mu \mathrm{L}$, flow rate: $7 \mu \mathrm{L} / \mathrm{min}$, time: $30 \mathrm{~min}$ ). After detection, the surface of the sensing chip could be regenerated with $50 \mathrm{mM} \mathrm{NaOH}$ (loading volume: $150 \mu \mathrm{L}$, flow rate: $20 \mu \mathrm{L} / \mathrm{min}$, time: $2 \mathrm{~min}$ ).

\section{Results and discussion Illustration of the SPR biosensing strategy}

Scheme 1 clearly showed the detection procedures of HER2-positive exosomes using the reformative TSA activated by target-induced MAB transition. Specifically, exosomes were extracted from the cell culture fluid or clinical samples by ultracentrifugation, and the SPR signal amplifier of AuNPs-Ty was prepared by conjugating the tyramine molecules to AuNPs. Meanwhile, the ingeniously designed MAB containing HER2 aptamer (green domain) and G4 (red domain) sequences was modified on the surface of the gold chip by Au-S bond. In the presence of HER2-positive exosomes, the MAB captured the exosomes by the interaction of aptamer and HER2 protein, which led to the exposure of G4. The unlocked G4 formed catalytically active G4-hemin with the assistance of hemin and $\mathrm{K}^{+}$. Subsequently, the activated G4-hemin prompted a large amount of AuNPs-Ty to in situ deposit on the surface of the exosomal membrane through the TSA process, resulting in the production of a greatly enhanced SPR signal. As a result, the developed SPR strategy achieved label-free, real-time, and highly specific detection of low-abundance HER2-positive exosomes.

\section{Characterization of the extracted exosomes, G4-hemin, and AuNPs-Ty}

First, the morphology and concentration of exosomes derived from SK-BR3 cells were characterized, and the results were shown in Additional file 1: Fig. S1. The TEM image disclosed that the exosomes were round vesicles with a clear double-layer membrane structure (Additional file 1: Fig. S1A). The NTA indicated that the original concentration of the exosomes was $2.75 \times 10^{9}$ particles $/ \mathrm{mL}$ with a size distribution ranging from 30 to $200 \mathrm{~nm}$ (Additional file 1: Fig. S1B). The above results confirmed the successful extraction of exosomes. Second, the combination of G4 and hemin was verified by UV-vis absorption spectroscopy. As we can learn from Additional file 1: Fig. S2, $260 \mathrm{~nm}$ and $394 \mathrm{~nm}$ were the characteristic absorption peaks of G4 and hemin, respectively. As expected, in the absorption spectrum of G4-hemin, the absorption peaks of the two substances with a slight right shift were observed at the same time, indicating the successful formation of G4-hemin. Third, the characterization 


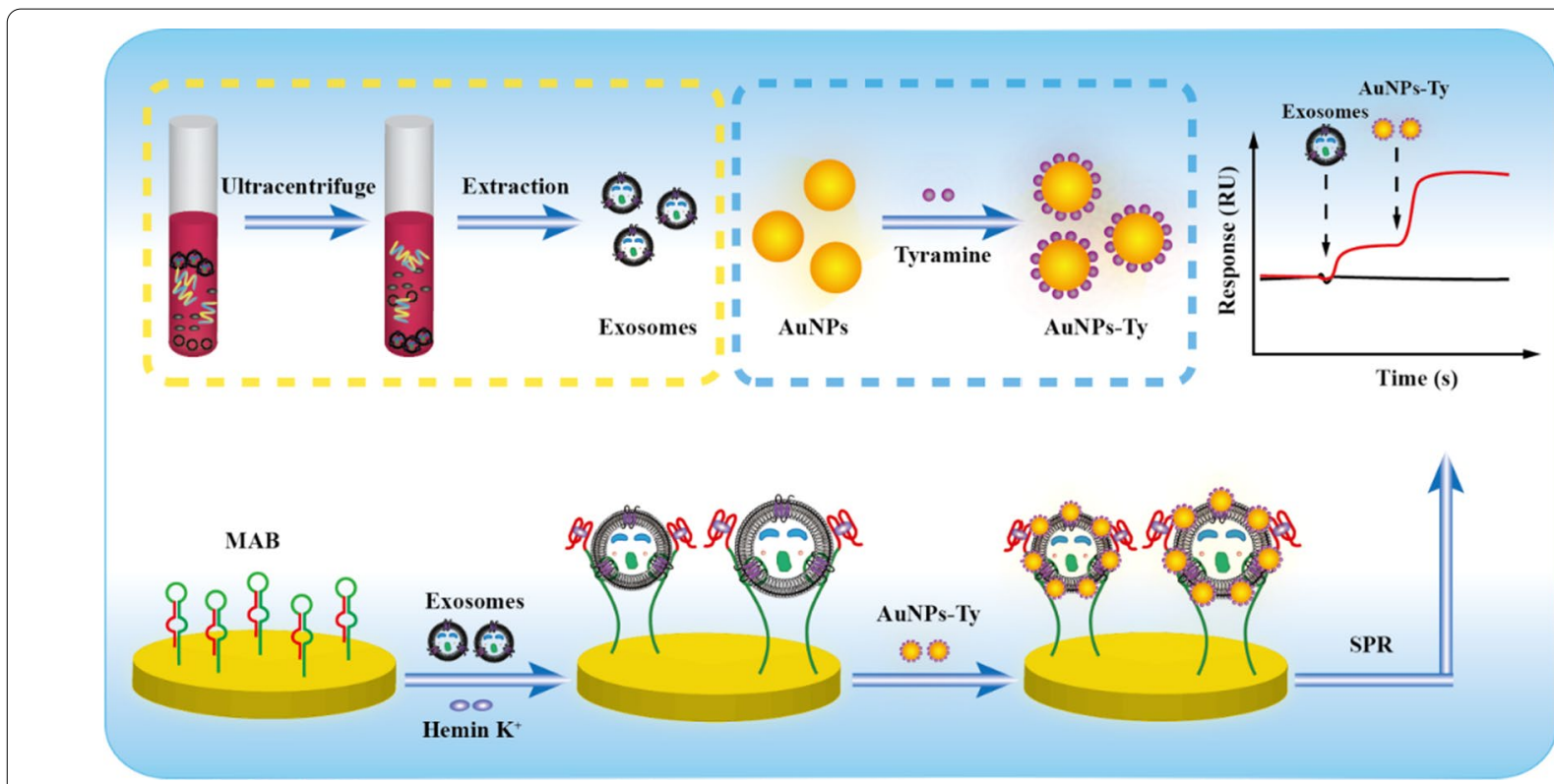

Scheme 1 Schematic illustration for the detection of HER2-positive exosomes based on the improved TSA enabled by target-induced MAB conversion
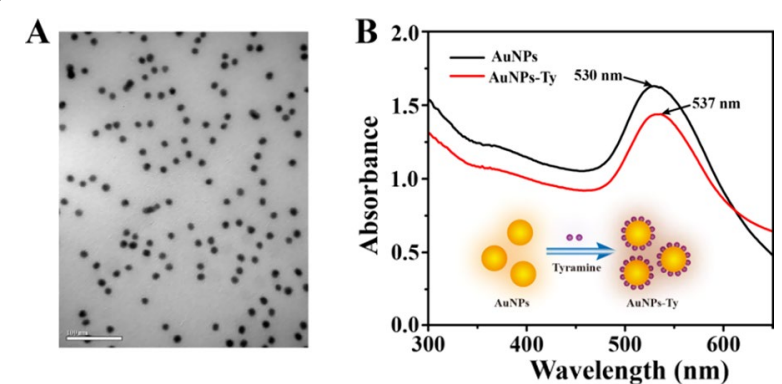

Fig. 1 Characterization of the signal amplifier of AuNPs-Ty. A TEM image of AuNPs. Scale bars $=100 \mathrm{~nm}$, B UV-vis absorption spectra of AuNPs (black line) and AuNPs-Ty (red line)

of AuNPs-Ty composite was performed. The TEM image showed the prepared AuNPs were uniform in volume and evenly distributed (Fig. 1A), and the UV-vis absorption spectra displayed that the typic absorption peaks of AuNPs and AuNPs-Ty were seen at $530 \mathrm{~nm}$ and $537 \mathrm{~nm}$, respectively (Fig. 1B). These results were entirely consistent with the previous report [24], indicating the successful synthesis of AuNPs-Ty.

\section{Feasibility of the developed biosensor}

To demonstrate the feasibility of this proposed biosensor, the whole detection process was firstly analyzed. The detection steps and the corresponding sensorgrams were depicted in Fig. 2A and B, respectively. In the first step, when the HER2-positive exosomes were loaded, the SPR signal rose by about $40 \mathrm{RU}$, indicating that the exosomes were connected to the surface of the chip owing to the effective binding of the aptamer of the MAB and HER2 proteins. In the second step, once the AuNPs-Ty composite was loaded, the SPR signal increased by about $200 \mathrm{RU}$, which was attributed to the deposition of plentiful AuNPs-Ty on the surface of the exosomal membrane caused by the reformative TSA. After detection, the chip surface was regenerated with $50 \mathrm{mM} \mathrm{NaOH}$ for next measurement. Subsequently, to verify that the deposition of AuNPs-Ty on the exosome surface was caused by the catalytic activity of G4-hemin rather than the nonspecific absorption, the relevant experiments were conducted. As shown in Fig. 2C, in the absence of hemin, the signal of the AuNPs-Ty was not been observed due to the fact that the exposed G4 had not peroxidase-like activity. In contrast, the AuNPs-Ty generated a distinct SPR signal, demonstrating that the signal indeed originated from the reformative TSA. In addition, compared with Ty and AuNPs that produced negligible SPR signal, the signal of AuNPs-Ty increased nearly tenfold (Fig. 2D). These results adequately demonstrated the feasibility of the proposed method for the detection of HER2-positive exosomes.

\section{Optimization of experimental parameters}

Several important reaction parameters of this biosensor were optimized to obtain good analytical performance in the presence of $1.0 \times 10^{7}$ particles/mL HER2-positive exosomes. Owing to the effect of the density of capture 
A
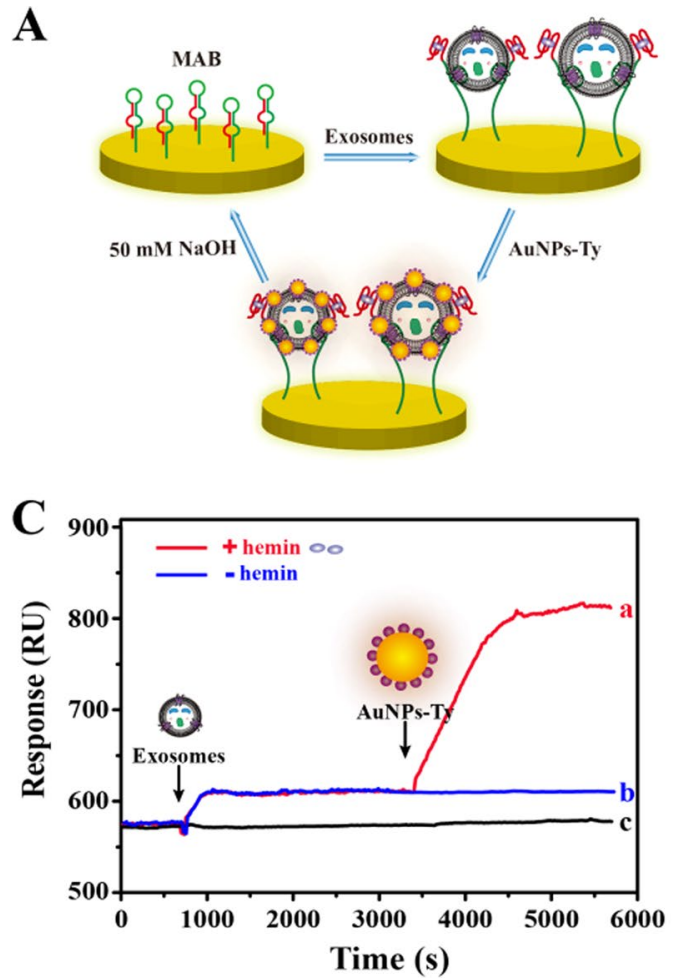
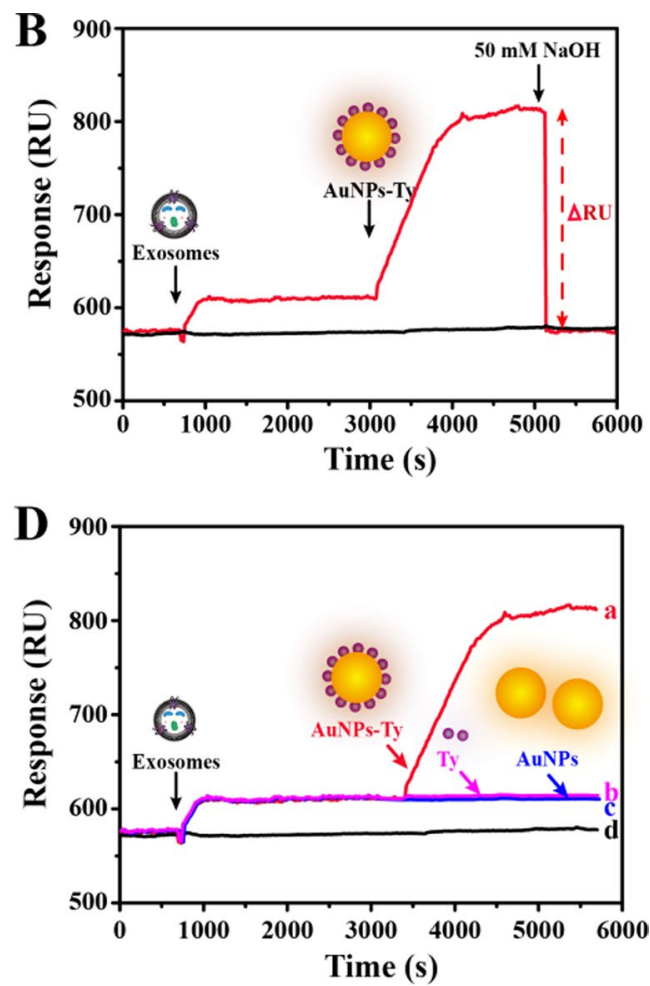

Fig. 2 Evaluation of the feasibility of the biosensing strategy. A Schematic diagram of the detection process. B Typical SPR sensorgrams of the biosensor (red line) and blank control without loading (black line). C SPR sensorgrams of the different conditions: (a) the intact sensing system, (b) the sensing system without hemin, and (c) blank control. D SPR sensorgrams of the different materials: (a) AuNPs-Ty, (b) Ty, (c) AuNPs, and (d) blank control. The concentration of HER2-positive exosomes derived from SK-BR3 cells is $1 \times 10^{7}$ particles $/ \mathrm{mL}$. All data expressed as mean \pm standard variation $(n=3)$

probe immobilized on the sensing chip on the sensitivity of the biosensor, the concentration of the MAB $\left(\mathrm{C}_{\mathrm{MAB}}\right)$ was firstly optimized. As shown in Additional file 1: Fig. S3A, the SPR signal gradually improved with the increase of the $\mathrm{C}_{\mathrm{MAB}}$ ranging from 0.5 to $1.5 \mu \mathrm{M}$. Once the concentration was above $1.5 \mu \mathrm{M}$, the signal decreased rapidly, because the crowded MAB could not bind to the exosomes effectively. Therefore, $1.5 \mu \mathrm{M}$ was chosen as the optimal $\mathrm{C}_{\mathrm{MAB}}$. When the concentration of hemin $\left(C_{\text {hemin }}\right)$ was $1.5 \mu \mathrm{M}$, being the same as the $C_{M A B}$, the highest signal was obtained. Hence, the optimal $\mathrm{C}_{\text {hemin }}$ is set to $1.5 \mu \mathrm{M}$ (Additional file 1: Fig. S3B).

Furthermore, to avoid the nonspecific deposition of redundant signal amplifier on the chip surface which increased the background signal, the befitting concentration of the AuNPs-Ty $\left(\mathrm{C}_{\text {AuNPs-Ty }}\right)$ was evaluated. As shown in Additional file 1: Fig. S3C, when the $\mathrm{C}_{\text {AuNPs-Ty }}$ rose from 50 to $200 \mathrm{mM}$, the SPR signal rose synchronously and ended by a downward trend. Therefore, we chose $200 \mathrm{mM}$ as the optimal $\mathrm{C}_{\text {AuNPs-Ty. }}$. Subsequently, we also optimized the reaction time between exosomes and MAB (Time ${ }_{1}$ ) and the incubation time of the improved TSA (Time ${ }_{2}$ ), respectively (Additional file 1: Fig. S3D and
E). When Time ${ }_{1}$ was set as $30 \mathrm{~min}$, the signal reached the peak and remained steady as time prolonged, indicating the completion of the reaction. Similarly, in the optimization experiment of $\mathrm{Time}_{2}$, the reaction was completed after $30 \mathrm{~min}$. Therefore, we set both reaction times as $30 \mathrm{~min}$.

\section{Sensitivity of the SPR biosensor}

Under the optimal reaction conditions, we further evaluated the sensitivity of this sensing method by detecting a series of different concentrations of HER2-positive exosomes. As shown in Fig. 3A, when the concentration of exosomes increased from 0.1 to $100 \times 10^{5}$ particles/ $\mathrm{mL}$, the SPR signal also increased simultaneously, showing a good correlation. As presented in, the regression equation between the concentration of exosomes $(\mathrm{X})$ and the corresponding SPR signals $(\mathrm{Y})$ was $\mathrm{Y}=2.36 \mathrm{X}+18.93$ with a correlation coefficient of 0.9914 (Fig. 3B). The lowest detectable concentration of the sensing strategy was $1.0 \times 10^{4}$ particles $/ \mathrm{mL}$, which was comparable to other biosensing strategies for exosomes detection, and the detailed comparison was shown in Additional file 1: Table S2. The excellent analytical performance of this 

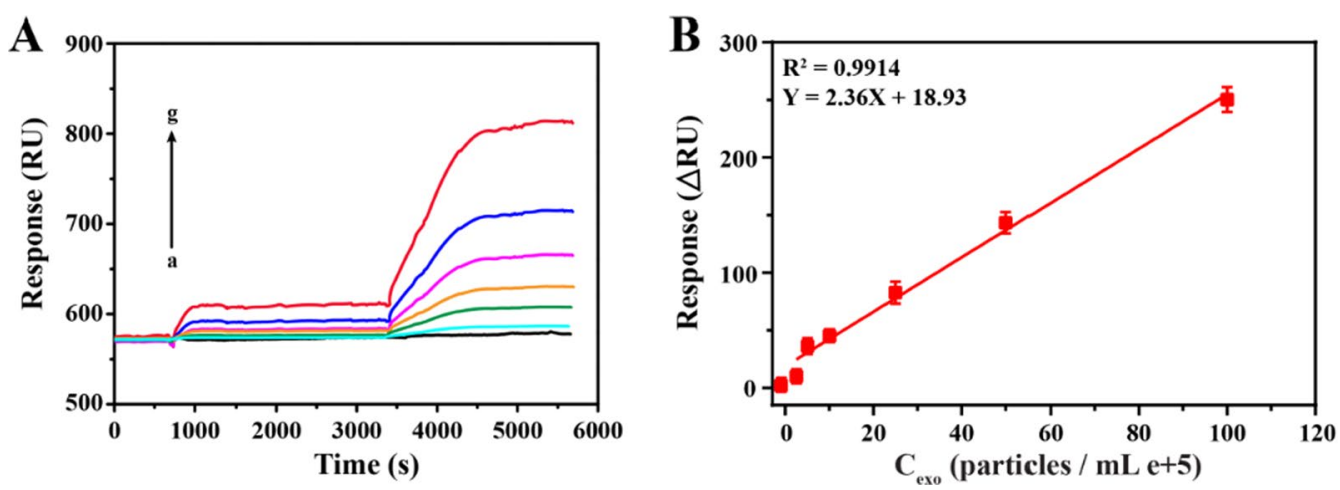

Fig. 3 Evaluation of the sensitivity of the SPR biosensor. A SPR sensorgrams and (B) the calibration line for exosomes at different concentrations of $0.1,2.5,5,10,25,50,100$ particles $/ \mathrm{mL}$ e +5 (from a to $\mathrm{g}$ ). All data expressed as mean \pm standard variation $(\mathrm{n}=3)$

method is mainly due to these aspects. First, the high affinity of the aptamer to HER2 proteins allowed the G4 sequence to be fully exposed to form the G4-hemin. Second, the formed G4-hemin exerted superior peroxidaselike activity itself to catalytic the in situ deposition of numerous AuNPs-Ty on the exosomes, resulting in the high SPR signal.

\section{Specificity of the SPR biosensing strategy}

The good specificity is one of the indispensable characteristics of an excellent biosensor, which directly affects the clinical application prospects of this method. Accordingly, we conducted follow-up researches to explore the specificity of this sensing strategy by choosing another four kinds of exosomes derived from different HER2negative cancer cells (HeLa, LNcap, HepG2, and MCF-7) as interferents. As shown in Fig. 4, compared with these interferents, exosomes from HER2-positive SK-BR3 cells produced a significantly improved SPR signal. In general, the SPR signal of HER2-positive exosomes was 4.9 to 6.4 times to those of interfering substances at the same concentration $\left(1.0 \times 10^{7}\right.$ particles $\left./ \mathrm{mL}\right)$. Besides, by detecting exosomes using the developed strategy, the cancer cells with high HER2 expression could be discriminated accurately. These results showed the excellent specificity of this method, which was mainly attributed to the dualidentification of HER2 proteins and the lipid membrane of exosomes.

\section{Detection of exosomes in clinical samples}

To evaluate the applicability of this method for the diagnosis of breast cancer, exosomes derived from real clinical samples were analyzed. We collected 16 serum samples from the First Affiliated Hospital of Chongqing Medical University. Among them, half of the samples were from HER2-positive breast cancer patients (P1-P8), and the rest were from healthy groups $(\mathrm{H} 1-\mathrm{H} 8)$, which served as testing control. As shown in Fig. 5A, almost all SPR
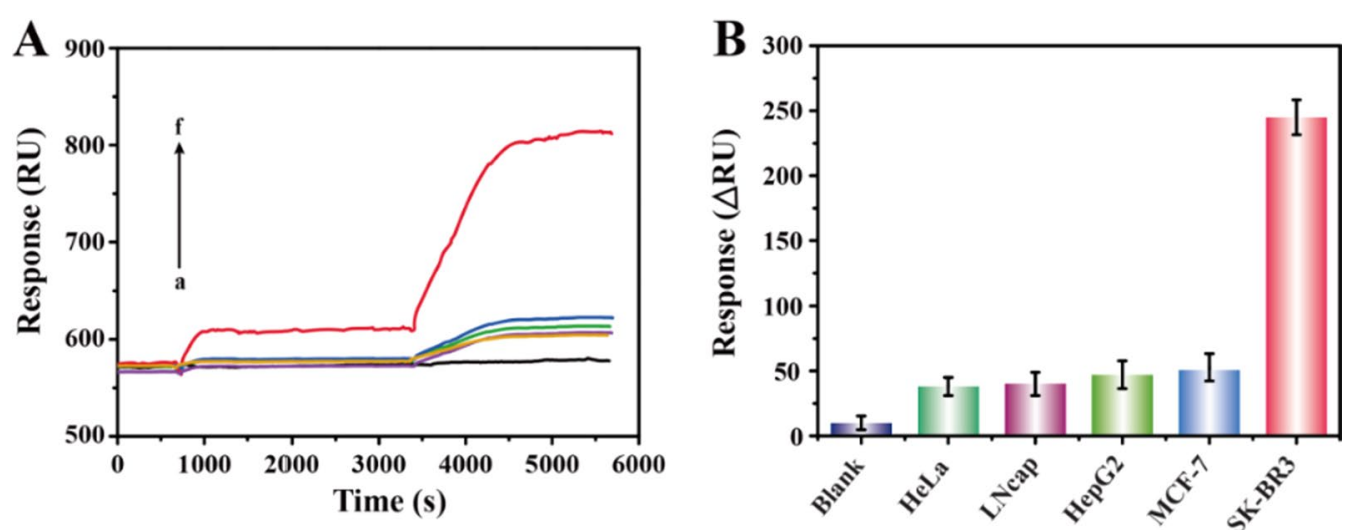

Fig. 4 Evaluation of the specificity of the sensing strategy. A SPR detection sensorgrams and (B) the response signals of (a) blank control and exosomes (1.0 × $10^{7}$ particles $/ \mathrm{mL}$ ) derived from (b) HeLa, (c) LNcap, (d) HepG2, (e) MCF-7, and (f) SK-BR3 cell lines, respectively. All data expressed as mean \pm standard variation $(n=3)$ 

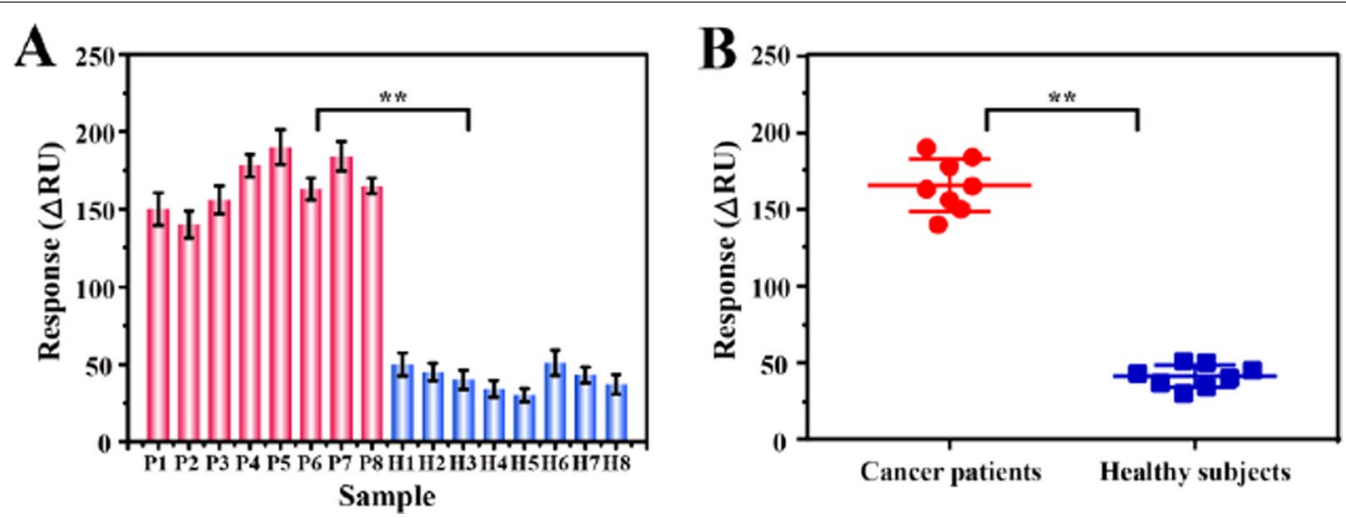

Fig. 5 Investigation of the clinical applicability of this biosensor. A Analysis of exosomes from clinical samples (breast cancer patients: P1-P8 and healthy control subjects: $\mathrm{H1}-\mathrm{H} 8$ ) by the developed strategy and (B) the corresponding scatter plot. Significance was determined by Student unpaired t-test, ${ }^{* *} p<0.05$

signals obtained by detecting exosomes from the serum of HER2-positive breast cancer patients were greater than 150 RU (except for P2), however, under the same circumstances, the signals obtained by detecting exosomes from healthy samples were all lower than 50 RU. The different signal levels of the positive samples were due to the fact that patients with different stages could secrete various concentrations of HER2-positive exosomes. In addition, the calculation of the significance probability $\mathrm{P}$-value also illustrated the significant difference between the two groups of data (Fig. 5B), implying the outstanding distinction ability of this method between HER2-positive breast cancer patients and healthy groups. These results shed light on the potential clinical application prospect of this sensing method.

\section{Conclusions}

In conclusion, a label-free SPR biosensor has been developed for highly sensitive and specific detection of HER2-positive exosomes based on the reformative TSA activated by target-induced MAB conversion. Different from the traditional TSA, the reformative TSA replacing HRP with G4-hemin fully avoids the intrinsic disadvantages of natural enzymes, thus possessing a high potential for clinical transformation. And the biosensing strategy is able to simultaneously recognize HER2 proteins and the lipid membrane of the exosomes, which effectively eliminates the interference of free proteins in purified exosomes. Benefiting from the integrating of the MAB and the reformative TSA, large quantities of AuNPs-Ty are in-situ deposited on the HER2-positive exosome membrane, endowing the developed SPR biosensor with high sensitivity and specificity. Moreover, by simply changing the aptamer types, this sensing strategy can be easily extended for accurate detection of other exosome subtypes. More importantly, this method has capable of discerning patients with HER2-positive breast cancer from healthy individuals. Overall, this work offers a new SPR platform for exosome-based liquid biopsy in the diagnosis of breast cancer. Despite these results, a limitation of this method is that the experimental steps need to be further simplified to improve the flexibility in clinical applications.

\section{Abbreviations}

TSA: Tyramine signal amplification; MAB: Molecular aptamer beacon; SPR: Surface plasmon resonance; AuNPs-Ty: Tyramine-coated AuNPs; HRP: Horseradish peroxidase; HER2: Human epidermal growth factor receptor 2; AuNPs: Gold nanoparticles; G4-hemin: G-quadruplex-hemin; MCH: 6-Mercapto-1-hexanol; FBS: Fetal Bovine Serum; DMEM: Dulbecco's Modified Eagle Medium; NHS: $\mathrm{N}$-hydroxysuccinimide; EDC: N-(3-(dimethylamino) propyl)-N'-ethylcarbodiimide hydrochloride; PBS: Phosphate buffer; RU: Resonance units; TEM: Transmission electron microscopic; NTA: Nanoparticles tracking analysis.

\section{Supplementary Information}

The online version contains supplementary material available at https://doi. org/10.1186/s12951-021-01210-x.

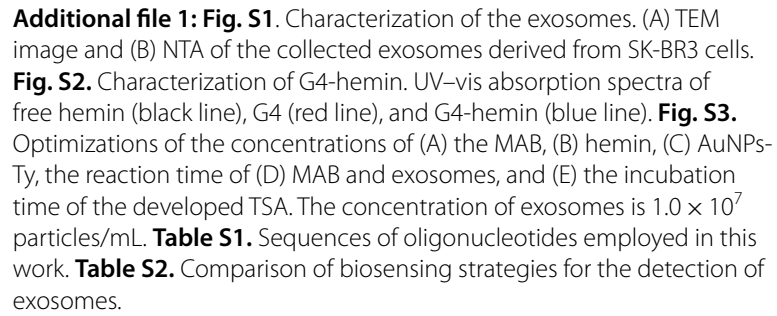
image and (B) NTA of the collected exosomes derived from SK-BR3 cells. Fig. S2. Characterization of G4-hemin. UV-vis absorption spectra of free hemin (black line), G4 (red line), and G4-hemin (blue line). Fig. S3. Optimizations of the concentrations of (A) the MAB, (B) hemin, (C) AuNPsTy, the reaction time of (D) MAB and exosomes, and (E) the incubation time of the developed TSA. The concentration of exosomes is $1.0 \times 10^{7}$ particles $/ \mathrm{mL}$. Table S1. Sequences of oligonucleotides employed in this work. Table S2. Comparison of biosensing strategies for the detection of exosomes.

\section{Acknowledgements}

This work was supported by the National Natural Science Foundation of China (81873980), the National Science and Technology Major Project of the Ministry of Science and Technology of China (2018ZX10732202), and the Science and Technology Project of the Health Planning Committee of Sichuan (19PJ159). 


\section{Authors' contributions}

WEC, ZL and WQC performed all experimental work. $J \mathrm{~L}, \mathrm{XL}$ and $\mathrm{LL}$ conducted data analysis. TW and HB collected clinical samples. SD and XL revised the manuscript and provided project guidance. $X Y$ procured funding and data curation. All authors read and approved the final manuscript.

\section{Availability of data and materials}

All data generated and analyzed during this study are included in this article and additional file. The additional file is available. Characterization of the exosomes, characterization of G4-hemin, and Optimizations of reaction conditions (Additional file 1: Figs. S1-S3). Sequences of oligonucleotides employed in this work (Additional file 1: Table S1). Comparison of biosensing strategies for the detection of exosomes (Additional file 1:Table S2).

\section{Declarations}

\section{Ethics approval and consent to participate}

This study has been approved by the ethics committee of Chongqing Medical University and conducted in accordance with ethical guidelines.

\section{Consent for publication}

All authors have provided consent for the manuscript to be published.

\section{Competing interests}

The authors declare that they have no competing interests.

\section{Author details}

${ }^{1}$ Key Laboratory of Clinical Laboratory Diagnostics (Ministry of Education), College of Laboratory Medicine, Chongqing Medical University, Chongqing 400016, China. ${ }^{2}$ Department of Clinical Laboratory, The Affiliated Drum Tower Hospital of Nanjing University Medical School, Nanjing 210008, China. ${ }^{3}$ Department of Laboratory Medicine, Zigong Fourth People's Hospital, Sichuan 643000, China.

Received: 17 October 2021 Accepted: 14 December 2021 Published online: 24 December 2021

\section{References}

1. Heer E, Harper A, Escandor N, Sung H, McCormack V, Fidler-Benaoudia M. Global burden and trends in premenopausal and postmenopausal breast cancer: a population-based study. Lancet Glob Health. 2020;8(8):e1027-37.

2. Li Y, Li Q, Mo H, Guan X, Lin S, Wang Z, Chen Y. Incidence, risk factors and survival of patients with brain metastases at initial metastatic breast cancer diagnosis in China. Breast. 2021;55:30-6.

3. Mitsogianni M, Trontzas I, Gomatou G, loannou S, Syrigos N, Kotteas E. The changing treatment of metastatic her2-positive breast cancer. Oncol Lett. 2021;21(4):287.

4. Semiglazova T, Sharashenidze S, Kerimova S, Klimenko V, Semiglazova V. Current approaches to the treatment of HER2 positive breast cancer with brain metastases. Tumors Female Reprod Syst. 2021;17(1):27-34.

5. Hassan E, Narjiss B, Mouna K, Abdelkader A, Meryem G. Safety of a trastuzumab biosimilar, used under routine clinical practice conditions in adult her $2+$ breast cancer patients in morocco. J Clin Oncol. 2020;38(15_suppl):e13024-e13024.

6. Zhang Y, Bi J, Huang J, Tang Y, Du S, Li P. Exosome: a review of its classification, isolation techniques, storage, diagnostic and targeted therapy applications. Int J Nanomed. 2020;15:6917-34.

7. Shi Y, Du L, Lv D, Li Y, Zhang Z, Huang X, Tang H. Emerging role and therapeutic application of exosome in hepatitis virus infection and associated diseases. J Gastroenterol. 2021;56(4):336-49.

8. Han C, Zhang C, Wang H, Zhao L. Exosome-mediated communication between tumor cells and tumor-associated macrophages: implications for tumor microenvironment. Oncoimmunology. 2021;10(1):1887552.

9. Fang S, Tian H, Li X, Jin D, Li X, Kong J, Yang C, Yang X. Clinical application of a microfluidic chip for immunocapture and quantification of circulating exosomes to assist breast cancer diagnosis and molecular classification. PLoS One. 2017;12(4):e0175050.

10. Kabe Y, Suematsu M, Sakamoto S, Hirai M, Koike I, Hishiki T, Matsuda A. Development of a highly sensitive device for counting the number of disease-specific exosomes in human sera. Clin Chem. 2018;64(10):1463-73.

11. Yan H, Li Y, Cheng S, Zeng Y. Advances in analytical technologies for extracellular vesicles. Anal Chem. 2021;93(11):4739-74.

12. Bagheri $\mathrm{H}$, Cha B, Lee E, Kim S, Park K. Advances in exosome analysis methods with an emphasis on electrochemistry. Anal Chem. 2020;92(19):12733-40.

13. Kim H, Oh C, An J, Baek S, Bock S, Kim J, Jung H, Song H, Kim J, Jo A Multi-quantum dots-embedded silica-encapsulated nanoparticlebased lateral flow assay for highly sensitive exosome detection. Nanomaterials. 2021;11(3):768.

14. Timson D. Myosin Va and spermine synthase: partners in exosome transport. Biosci Rep. 2019;39(4):BSR20190326.

15. Zhang Y, Wei Y, Liu P, Zhang $X$, Xu Z, Tan X, Chen M, Wang J. ICPMS and photothermal dual-readout assay for ultrasensitive and point-of-care detection of pancreatic cancer exosomes. Anal Chem. 2021;93(33):11540-6.

16. Zhang $X$, Liu M, He M, Chen S, Yu Y, Wang J. Integral multielement signals by DNA-programmed UCNP-AuNP nanosatellite assemblies for ultrasensitive ICP-MS detection of exosomal proteins and cancer identification. Anal Chem. 2021;93(16):6437-45.

17. Meng Y, Asghari M, Aslan M, Yilmaz A, Mateescu B, Stavrakis S, Demello A. Microfluidics for extracellular vesicle separation and mimetic synthesis: recent advances and future perspectives. Chem Eng J. 2021;404:126110.

18. Chen W, Li J, Wei X, Fan Y, Qian H, Li S, Xiang Y, Ding S. Surface plasmon resonance biosensor using hydrogel-AuNP supramolecular spheres for determination of prostate cancer-derived exosomes. Microchim Acta. 2020;187(11):590.

19. Wu W, Yu X, Wu J, Wu T, Fan Y, Chen W, Zhao M, Wu H, Li X, Ding S. Surface plasmon resonance imaging-based biosensor for multiplex and ultrasensitive detection of NSCLC-associated exosomal miRNAs using DNA programmed heterostructure of Au-on-Ag. Biosens Bioelectron. 2021;175:112835.

20. Dopie J, Sweredoski M, Moradian A, Belmont A. Tyramide signal amplification mass spectrometry (TSA-MS) ratio identifies nuclear speckle proteins. J Cell Biol. 2020;219(9):e201910207.

21. Zong $C$, Jiang F, Wang $X$, Li P, Xu L, Yang H. Imaging sensor array coupled with dual-signal amplification strategy for ultrasensitive chemiluminescence immunoassay of multiple mycotoxins. Biosens Bioelectron. 2021;177:112998.

22. Fu C, Jin S, Shi W, Oh J, Cao H, Jung Y. Catalyzed deposition of signal reporter for highly sensitive surface-enhanced raman spectroscopy immunoassay based on tyramine signal amplification strategy. Anal Chem. 2018;90(22):13159-62.

23. Zhou X, Li Y, Wu H, Huang W, Ju H, Ding S. A amperometric immunosensor for sensitive detection of circulating tumor cells using a tyramide signal amplification-based signal enhancement system. Biosens Bioelectron. 2019;130:88-94.

24. Kou B, Chai Y, Yuan Y, Yuan R. A DNA nanopillar as a scaffold to regulate the ratio and distance of mimic enzymes for an efficient cascade catalytic platform. Chem Sci. 2020;12(1):407-11.

25. Li J, Yuan T, Yang T, Xu L, Zhang L, Li H, Cheng W, Ding S. DNA-grafted hemin with preferable catalytic properties than G-quadruplex/hemin for fluorescent miRNA biosensing. Sens Actuators B. 2018;271:239-46.

26. Kouzegaran JV, Farhadi K, Forough M, Bahram M, Çetinkol PÖ. Highly-sensitive and fast detection of human telomeric G-Quadruplex DNA based on a hemin-conjugated fluorescent metal-organic framework platform. Biosens Bioelectron. 2021;178:112999.

27. Bakhtiar R. Surface plasmon resonance spectroscopy: a versatile technique in a biochemist's toolbox. J Chem Edu. 2013;90(2):203-9.

28. Das J, Kelley S. High-performance nucleic acid sensors for liquid biopsy applications. Angew Chem Int Ed Engl. 2020;59(7):2554-64.

29. Digehsaraei S, Salouti M, Amini B, Mahmazi S, Kalantari M, Kazemizadeh A, Mehrvand J. Developing a fluorescence immunosensor for detection of her2-positive breast cancer based on graphene and magnetic nanoparticles. Microchem J. 2021;167(1):106300. 
30. Xu H, Aguilar Z, Yang L, Kuang M, Duan H, Xiong Y, Wei H, Wang A. Antibody conjugated magnetic iron oxide nanoparticles for cancer cell separation in fresh whole blood. Biomaterials. 2011;32(36):9758-65.

31. Feng C, Zhou L, Yu T, Xu G, Tian H, Xu J, Xu H, Luo K. A new anticancer compound, oblongifolin $\mathrm{C}$, inhibits tumor growth and promotes apoptosis in HeLa cells through Bax activation. Int J Cancer. 2012;131 (6):1445-54.

32. Dahl M, Bouchelouche P, Kramer-Marek G, Capala J, Nordling J, Bouchelouche K. Sarcosine induces increase in HER2/neu expression in androgen-dependent prostate cancer cells. Mol Biol Rep. 2011;38(7):4237-43.

33. Liu J, Zhu L, Wang L, Chen Y, Giri B, Li J, Cheng G. Isolation and characterization of extracellular vesicles from adult Schistosoma japonicum. J Vis Exp. 2018;22(135):57514.

34. Ramírez-Bajo M, Banon-Maneus E, Rovira J, Campistol J, Diekmann F. Isolation of extracellular vesicles derived from mesenchymal stromal cells by ultracentrifugation. Bio Protoc. 2020;10(24):e3860.

35. Gen R, Ortiz M, O'Sullivan C. Diffusion-controlled synthesis of gold nanoparticles: nano-liposomes as mass transfer barrier. J Nanoparticle Res. 2014;16(4):1-5.

36. Manikandan M, Nasser Abdelhamid H, Talib A, Wu H. Facile synthesis of gold nanohexagons on graphene templates in raman spectroscopy for biosensing cancer and cancer stem cells. Biosens Bioelectron. 2014;15(55):180-6.

37. Abdelhamid H, Wu H. Gold nanoparticles assisted laser desorption/ ionization mass spectrometry and applications: from simple molecules to intact cells. Anal Bioanal Chem. 2016;408(17):4485-502.

38. Gopal J, Abdelhamid H, Huang J. Nondestructive detection of the freshness of fruits and vegetables using gold and silver nanoparticle mediated graphene enhanced Raman spectroscopy. Sens Actuators B. 2016;224:413-24.

39. Nguyen P, Zhang X, Su J. One-step controlled synthesis of size-tunable toroidal gold particles for biochemical sensing. ACS Appl Nano Mater. 2019;2(12):7839-47.

\section{Publisher's Note}

Springer Nature remains neutral with regard to jurisdictional claims in published maps and institutional affiliations.

Ready to submit your research? Choose BMC and benefit from:

- fast, convenient online submission

- thorough peer review by experienced researchers in your field

- rapid publication on acceptance

- support for research data, including large and complex data types

- gold Open Access which fosters wider collaboration and increased citations

- maximum visibility for your research: over $100 \mathrm{M}$ website views per year

At BMC, research is always in progress.

Learn more biomedcentral.com/submissions 